widely held belief, the total duration of a baby's sleep does not change up to the twelfth week, but that the day fraction of sleep, which is the smaller from birth, decreases. During the succeeding fourteen weeks the duration of night sleep remains un. changed; but day sleep decreases further until an adjustment to the infant's environment is reached; this consists in one long unbroken period of night sleep and short morning and afternoon naps. H. R. Richter (Basle) stated that sleep and wakefulness, like youth and age, entail different brain rhythms, a fact which can be used for the investigation of sleep. A. W. Eiff (Bonn) succeeded, by altering the clock during a short period of anæsthesia, not only in deceiving the time-sense of people living in a constant-condition room, but also found a shift in certain diurnal body rhythms, though not in others. D. Lüderitz (Münster) showed that thyrotoxic and myxœdematous patients differ from healthy people in their resistance to sleep-disturbing noises. T. Möllerström and S. Svensson (Stockholm) described the diurnal rhythms of various types of diabetes and stressed the success of the adjustment of therapeutic measures to those rhythms. In a more general way this principle was supported by W. Menzel and A. Jores (Hamburg).

G. Metz and P. Andlauer (Strasbourg) reported that in certain selected industries fewer accidents occurred during night shifts than during day shifts, while A. Pierach (Nauheim) described the adverse effects of night work and of frequent changes of working hours, especially on diseased people.

It is hoped that-as was the case after the last conference - the papers and discussions of this conference will be published in a supplement to the Acta Medica Scandinavica. Profs. A. Jores and W. Menzel, of Hamburg (2. Medizinische Klinilk), were elected president and secretary of the Society. A sub-committee on terminology was also elected. The next conference will be held in Stockholm in 1955 .

H. Katmus

\section{ORGANO-PHOSPHORUS INSECTICIDES}

A

SYMPOSIUM on organo-phosphorus insecticides, arranged jointly by the Crop Protection Panel of the Society of Chemical Industry, the Association of Applied Biologists, the Biological Methods Group of the Society of Public Analysts and Other Analytical Chemists and the Pharmacological Society, was held at the Imperial College of Science and Technology, London, on the morning and afternoon of October 2. The meeting attracted a large audience, more than filling the large Chemistry Theatre of the College.

The large attendance may have owed more to the participation of four learned societies than to the choice of a topical subject which is much in the scientific news, because the open discussion was not as lively as one might have expected, only a small proportion of those present participating. Coming so soon after the much more active two-day discussion on "Anti-cholinesterases", organized by the British Pharmacological Society and the Society of Chemical Industry, which was held during March 27-28, one wonders whether the subject is not a little 'played out'. A more likely explanation, how- ever, of the somewhat apathetic discussion may have arisen from the fact that so many societies were interested. One feels that their representatives on the joint committee may have been too polite to press their own interests, with the result that the five invited lecturers presented a rather incoherent display, and the audience was left wondering what were the most important arguments in which they were expected to participate.

There is unquestionable good in different societies coming together for such symposia, but it is more important than when they meet alone that the subject-matter should be selected and restricted, and a clear field of controversy mapped out. Granted this greater care of planning, which is clearly a more difficult task for an ad hoc joint committee, meetings of the most diverse societies can be very successful. Where this is lacking, there tends to be a diffidence on the part of the various contributors to discuss the particular aspects of the most interest to them in the presence of representatives of other professions, who they feel may not be particularly interested. It was this factor, I think, which tended to discourage the discussion on this occasion.

The morning session was devoted to toxicology : a paper prepared by Dr. K. A. Lord and Dr. C. Potter, of the Rothamsted Experimental Station, was read by the former, dealing with the insect aspect, and one by Dr. J. M. Barnes, of the Medical Research Council Toxicology Research Unit, Carshalton, dealing with the mammalian aspect. Prof. V. B. Wigglesworth (Cambridge) took the chair.

Prof. Wigglesworth spoke of the importance of biological interactions in the control of insect pests and the importance, therefore, of species-selective insecticides, as well as of the necessity for selectivity of a wider kind in the choice and development of insecticides less poisonous to man. He emphasized that a great deal is still to be learned about the biochemistry of selectivity. The systemic insecticide 'Schradan' is highly selective, but the mechanism is far from clear. Phytophagous caterpillars are notoriously immune, and, although they possess an unusually alkaline gut content, this can scarcely account for rendering innocuous a substance very resistant to alkaline hydrolysis. Something much more subtle is involved. Systemic insecticides also pose new problems in the matter of safety to man, since an insect poison incorporated into the structure of the plant is something new.

Dr. Lord said that, although the function of acetylcholine in myoneural transmission in mammals is well established and the toxic action of the organophosphorus compounds is undoubtedly mainly due to their inhibition of cholinesterase, even the existence of acetylcholine is not clearly established in insects, and it is far from certain that it has any important part to play. He felt, therefore, that the scope of investigation in insects should be widened to include other esterases which are also inhibited by the organo-phosphorus compounds. Crude extracts from insects have considerable activity in hydrolysing phenyl acetate, which has been used as a substrate in many experiments.

No general correlation has been established between insecticidal activity of various compounds and their ability to inhibit either cholinesterase or the general esterase in vitro. This is doubtless due to prior conversion of the compound in vivo as in the case of pure 'Parathion', which does not inhibit either esterase in vitro but is converted in Tenebrio molitor 
to a compound which does. In other cases lack of correlation may be due to physical factors, such as membrane permeability and partitioning between aqueous and lipoid phases, and it must be remembered that the enzymes are localized in vivo and that this localization is necessarily lost in making a crude extract.

Some organo-phosphorus compounds are toxic to insect eggs, although insufficiently in most cases to be of practical importance. Extracts of tomato moth eggs do not hydrolyse acetylcholine unless made from eggs in a late stage of development. Extracts from all stages hydrolyse phenyl acetate. Tetraethyl pyrophosphate ('IEPP) at high concentration kills eggs at all stages, suggesting that the toxic action is exerted via the general-rather than the cholineesterase.

Dr. Barnes divided his description of the information on organo-phosphorus poisons on mammals into three parts: first, the acute effects and death; second, long-term and chronic effects; and third, the inhibition of cholinesterase. All cases of death are finally directly due to inhibition of respiration caused by the failure of neuro-muscular transmission, in most cases at both ends of the nervous system; but it is to be noted that some compounds, for example, 'Schradan', produce very little effect on the brain cholinesterase at the time of death, and in such cases failure of transmission may be confined to the muscle end of the nerve system. Fatal outcome can in many cases be avoided by application of artificial respiration until the internal mechanism of respiration has recovered, but the success of this depends on the reversibility of the cholinesterase inhibition. Different compounds differ in this respect ; for example, among the phosphoric esters with an active group, those where the stable groups are methoxy are more easily reversed than where they are ethoxy, which in turn are very much more easily reversed than where the isopoproxy is concerned; and, in fact, artificial respiration fails to produce recovery after intoxication by di-isopropyl from the phosphonate.

In describing the long-term and chronic effects, Dr. Barnes again directed attention to the importance of reversibility, and mentioned also the prolonged paralytic effects resulting as a delayed action after severe intoxication with some compounds. This delayed action, which has operated in man, has not done so in the smaller mammals but can easily be experimentally demonstrated in birds. Unfortunately, the common factor among the compounds which produce this effect cannot be indicated, and so the probability of its occurrence must be determined entirely empirically.

In the case of mammals there is no doubt that the inhibition of cholinesterase is primarily responsible for the external symptoms; but as with insects, though in less degree, other esterases may be involved, and in fact it has been known for some time that the pseudo cholinesterase of serum is more effective in hydrolysing some other esters of choline than acetylcholine itself.

Dr. B. A. Kilby, of the School of Medicine, University of Leeds, opening the discussion, said that the mystery of 'Schradan'-a toxic substance not inhibiting in vitro-has been largely cleared up, oxidation to the amide oxide, an inhibiting and easily hydrolysed intermediate, being responsible. He felt that we should not be too afraid of compounds of high initial toxicity if they are quickly destroyed, and wondered whether the time has not come when we could contemplate the use of more toxic rather than less toxic insecticides.

During the discussion which followed, Dr. P. P. Hopf described some work in which the specific action of organo-phosphorus compounds against different enzymes appears very clearly. $\mathrm{He}$ has found, in working with diethyl phenyl phosphates, that the compound with chlorine substituted in the ortho position is very much more effective against the general esterase, while that with chlorine substituted in the para position is much more effective against cholinesterase. At a later stage of the discussion, Dr. W. N. Aldridge emphasized the extreme importance of chemical purity when examining the effect of organo-phosphorus compounds on enzymes in vitro, when there is a possibility of the compound under investigation being contaminated with a very much more active one. For example, esters of diethyl phosphoric acid are frequently contaminated with the extremely active inhibitor, tetraethyl pyrophosphate.

Dr. D. F. Heath pointed out that many of the reversible anti-cholinesterases depend for their absorption on the precise geometrical fit of two active centres in the molecule, and their specificity for different enzymes could doubtless be due to this stereochemical factor; whereas the majority of the organo-phosphorus inhibitors become covalently linked to one active centre in the enzyme, involving disruption of a link in the original compound. In this case, a lower degree of selectivity is to be expected, arising from modification of reaction-rates, influenced rather less drastically by stereochemical factors.

Taking up Dr. Kilby's query about the use of more highly toxic compounds, Dr. E. F. Edson discussed the human safety aspect. He emphasized that the relative toxicity to mammals and insects is important, and the new insecticide 'Malathon', having about a hundredth the toxicity of 'Parathion' to mammals and about a quarter of its toxicity to the majority of insects, can be regarded, therefore, as having a twenty-five-fold factor of safety in use. He was particularly concerned with the volatility of toxic compounds in regard to the risks of their use in the field, because the obvious protective measure, namely, the respirator, is an uncomfortable device under hot, sunny conditions, and workers are very inclined to evade its use. He mentioned that the safety aspects of 'Schradan' have been very fully investigated. His own information on rats and pigs indicates that a daily intake of about one twohundredth of a lethal dose produces scarcely any detectable effect. Alone among the insecticides, this compound has been used to a considerable extent for therapeutical purposes (notably by Ryder and DuBois in the United States) in treatment of myas. thenia gravis, and, although doses have been used requiring the additional use of atropine, many patients have been able to return to normal activities.

The afternoon session was concerned mainly with analytical aspects, under the chairmanship of Dr. J. R. Nicholls. Dr. G. S. Hartley, of Pest Control, Ltd., dealt with the behaviour of 'Schradan' in the living plant. Several lines of evidence lead clearly to the conclusion that the biochemical attack is in the first place an oxidation of a peripheral group in the molecule, and that it is probably basically similar to the oxidation occurring in the mammalian liver, but the conversion is very much slower. Oxidation of 'Schradan' in inanimate systems has also been 
examined, and recently Spencer and O'Brien in Canada have examined the effect of partial chlorination in anhydrous systems. Oxidation always produces an active inhibitor of cholinesterase, but one which is very unstable in water and leads to complete inactivation of the molecule by hydrolysis or partial conversion to a less active compound. Many steps in the oxidation are possible, as in the case of oxidation of simple tertiary amines, and the whole sequence of chemical processes is likely to be complex; but all the evidence points to each successive step of oxidation producing a compound yet more unstable than the last.

The amount of active inhibitor present during vegetable decomposition is always extremely small and follows closely the concentration of the reservoir of unchanged 'Schradan'. Were the observed inhibitions due to the much more toxic compound tetraethyl pyrophosphate, they are so small that they would be considered to be quite without toxic significance. The susceptible animal converts 'Schradan' to an inhibitor far more rapidly than the plant, and therefore the transient inhibitor attains much higher concentrations. It is therefore quite without importance whether a very small amount of conversion has previously occurred within the plant.

Dr. Hartley described some of the more recent work done by Dr. Heath and collaborators, which has unearthed a rather more stable vegetable derivative of 'Schradan' that is not itself an activator. The generally accepted view is that the active derivative has an amide oxide structure. The less active and more stable derivative has probably a hydroxymethyl group. He felt that the decomposition of 'Schradan' inside the plant has now been sufficiently explored to meet any reasonable doubts about the safety of this compound. Questions are still being asked as to whether the plant might not resynthesize some quite new toxic substance from the original insecticide, but he pointed out that there are many naturally toxic compounds in food crops, and an equal anxiety should really arise, therefore, with regard to the activities of the plant breeder and the applicant of fertilizers.

Dr. P. R. Carter, of Albright and Wilson, Ltd., gave a detailed account of the application of measurements of differential hydrolysis-rate to the analysis of mixtures of organo-phosphorus compounds.

Mr. J. F. Newman, of Imperial Chemical Industries, Ltd., then dealt with the technique of bioassay of the organo-phosphorus compounds. He said that chemical methods are always to be preferred for precise determination in cases where there is no doubt about the compound to be determined; but in many cases one does not know the full story about the chemical composition in relation to toxic effect, either because the original compounds are associated with active impurity, or because there may have been some conversion as a result of biochemical or weathering attack after application. Biological assay has therefore a necessary part to play, and it is also in the majority of cases capable of greater sensitivity, although of less accuracy than chemical methods. Mr. Newman described various devices for rendering biological assay methods more reproducible, and dealt with the importance of choosing the most suitable measure of intoxication, which is very rarely death itself. He said that he prefers, where possible, to use aquatic species, such as mosquito larvæ or daphnia, so that the toxic substance can be presented in a uniform environment.
There was general agreement with Mr. Newman's thesis that bioassay methods are absolutely necessary in many cases, although never so precise as chemical methods, and Mr. A. L. Bacharach pointed out that the development of pharmaceutical science owes a very great deal to the alternate use and comparison of biological and chemical methods of estimation.

Dr. Edson made a plea for a bolder approach on the part of the medical profession to the problem of permissible residues of toxic materials. The reluctance of medical workers to commit themselves as to what can be permitted as safe is doubtless due to the inherent difficulty of proving a negative. It is so much easier to prove that a large amount is dangerous than to prove that a small amount is safe; but he felt that the importance of the practical problem demands that people should be bold enough to make decisions, even at the risk of their being proved wrong by later generations. Dr. R. A. E. Galley supported this very realistic attitude, and quoted a remark of a distinguished contributor to this subject, that there is more danger of men's minds being poisoned against the use of insecticides than there is of their bodies being poisoned by the insecticides themselves.

G. S. HARTLEY

\section{BRIGHTON AND HOVE NATURAL HISTORY SOCIETY \\ CENTENARY SESSION}

$T$ HE Brighton and Hove Natural History Society will attain its centenary during the present session, which opened on October 10 with a review of the past hundred years by the general secretary, Mr. F. Edwards, followed by a conversazione.

The exact date of the founding of the Society is not known with certainty; but it was no doubt a natural development of some of the existing societies such as the Royal Literary Society. The proceedings at the early meetings, with the discussion thereon, were recorded in manuscript and consisted of papers by members; specimens of plants, fossils, etc., were often exhibited. An annual excursion to some place of interest was a regular feature which survived many years, and the earlier excursions were followed by a paper by the secretary, or other member, describing the features of interest observed, often including local legends and ancient history ; sketches by a member have been preserved in a portfolio.

During the early period the papers read were all due to the members themselves, and, although later on non-members were invited to give addresses, it is only of recent years that the greater number of papers have been given by non-members. Summaries of the papers were given in the annual reports, and from about 1873 many of them were printed in full. In those days, also, the local newspapers often printed the transactions at the meetings in full, a contrast to the present day when a mention of the meetings is a rare occurrence.

The principal function of a society of this nature is the mutual exchange of scientific knowledge between the members themselves; but its activities should go beyond this, and it is expected that it should either make contributions to science by original investigations by members, or should 